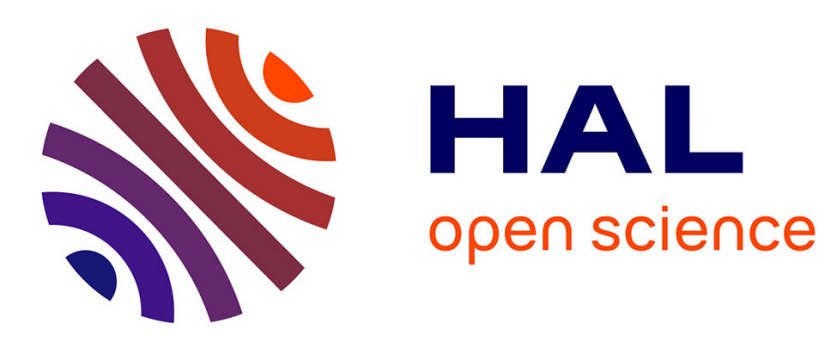

\title{
Microsecond infrared beam bending in photorefractive iron doped indium phosphide
}

\author{
Nicolas Fressengeas, Cristian Dan, Delphine Wolfersberger
}

\section{To cite this version:}

Nicolas Fressengeas, Cristian Dan, Delphine Wolfersberger. Microsecond infrared beam bending in photorefractive iron doped indium phosphide. Optics and Laser Technology, 2013, 48, pp.96-101. 10.1016/j.optlastec.2012.09.018 . hal-00751970

\section{HAL Id: hal-00751970 \\ https://hal.science/hal-00751970}

Submitted on 14 Nov 2012

HAL is a multi-disciplinary open access archive for the deposit and dissemination of scientific research documents, whether they are published or not. The documents may come from teaching and research institutions in France or abroad, or from public or private research centers.
L'archive ouverte pluridisciplinaire HAL, est destinée au dépôt et à la diffusion de documents scientifiques de niveau recherche, publiés ou non, émanant des établissements d'enseignement et de recherche français ou étrangers, des laboratoires publics ou privés. 


\title{
Micro-second infra-red beam bending in photorefractive Iron doped Indium Phosphide
}

\author{
N. Fressengeas ${ }^{\mathrm{a}}$, C. Dan ${ }^{\mathrm{a}, \mathrm{b}}$, D. Wolfersberger ${ }^{\mathrm{b}}$ \\ ${ }^{a}$ Université de Lorraine, Laboratoire Matériaux Optiques, Photonique et Systèmes (EA 4423), Metz, F-57070, France \\ ${ }^{b}$ Supélec, Laboratoire Matériaux Optiques, Photonique et Systèmes (EA 4423), Metz, F-57070, France
}

\begin{abstract}
A time resolved study of the behavior of a single beam in photorefractive iron doped indium phosphide is provided down to the microsecond range, showing that infrared beam bending does occur on the micro-second time scale for moderate beam intensities. Two distinct time scales are evidenced, the behavior of which are the sign of two different photorefractive mechanisms.
\end{abstract}

\section{Introduction}

Self focusing of a laser beam in a photorefractive (PR) materials is a process that leads to the formation of waveguides in bulk crystals. Under the right conditions, such a self focused beam can propagate as a spatial soliton $[1,2]$. Since their first observation [3, 4], the PR solitons have been modeled and the models found their experimental validations. As such, their properties are well known in typical PR materials (such as SBN, $\mathrm{Bi}_{12} \mathrm{TiO}_{20}$ and $\mathrm{BaTiO}_{3}$ [5-8]), in which the PR effect is due mainly to only one type of charge carriers and occurs at visible wavelengths: the dynamics of self focusing and soliton formation have been studied and characterized at steady state [9-12] as well as in transient regime $[13,14]$.

However, all the materials that obey this one carrier model have somewhat slow dynamics: the fastest of them, tin hypothiodiphosphate $\left(\mathrm{Sn}_{2} \mathrm{P}_{2} \mathrm{~S}_{6}\right)$ has time reactions above ten milliseconds for intensities above $100 \mathrm{~W} / \mathrm{cm}^{2}$ [15]. Obtaining a photorefractive self-focused beam in a shorter time requires either going to really high beam intensities $\left(\mathrm{MW} / \mathrm{cm}^{2}\right)[16]$ or change the material type towards semi-conductors such as iron doped Indium Phosphide [17] where micro-second response times can be obtained for intensities as low as a $\mathrm{W} / \mathrm{cm}^{2}$, paving the way for information all-optical routing in telecommunication networks.

Indeed, as was shown previously[18], two self-focused beams can interact with each other in a non linear way, literally colliding with each other. Therefore, if fast enough build-up is achieved, an all optical beam steering device can be based upon the collision of a rapidly modulated signal beam and a control beam. The resulting device would be transparent to high frequency signal modulation and would allow beam steering with no moving part.

This particular light configuration, consisting of two distinct beams on a dark background is essentially non periodic and high contrast, while two-carrier-photorefractivity has been studied and understood two decades ago[19-22] in the framework of periodic illumination only. Its behavior under low non-periodic illumination has been inferred from the previous works[23]. However, its precise behavior under high contrast non-periodic illumination still remains to be fully understood, building on a partial previous model [24], full 3D numerical simulations [25] and steady-state experiments [26].

In this paper, a time resolved experimental analysis of a single beam bending behavior in a biased photorefractive InP:Fe sample is proposed for two different wavelengths. One beam, of wavelength either 1.06 or 1.55 microns, is shone on an iron doped indium phosphide sample and its behavior is analyzed in a time resolved fashion below the micro-second range. The best solution for this analysis would be to make a movie imaging the beam on the crystal output face, using an infra-red high speed camera. However, as we aim to a time resolution below the microsecond, this solution is very expensive. We have thus devised a cheaper, yet insightful, method based on a position detector. In the following sections, the experimental apparatus will be detailed, followed by measures as functions of beam intensity, background homogeneous intensity and temperature, for the two wavelengths whenever possible.

\section{Experimental setup}

The experimental set-up we used is similar to one that was previously published [26]: a Gaussian free space laser beam either issued from a $1064 \mathrm{~nm} \mathrm{CW}$ Nd: YAG laser or from a Continuous Wave (CW) $1.55 \mu \mathrm{m}$ free space collimated Laser Diode is spatially filtered and switched on and off using an accousto-optic modulator. Two different accousto-optic modulators were used for the two wavelengths, both have a rise time of 500ns. The laser beam is focused down to a $25 \mu \mathrm{m}$ waist on the entrance face of a $10^{17}$ doped InP:Fe sample, as measured from Secondary 
(a)

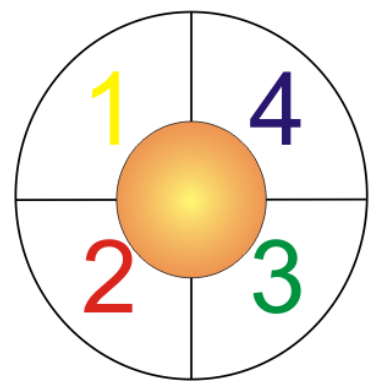

Figure 1: The position detector four quadrants. When the electric field is off, the beam is centered (a). When the field is switched on, the beam is no longer centered, because of beam-bending, and can be self-focused (b).

Ion Mass Spectroscopy. As in a previous experiment [26], the InP sample has dimensions $10 \times 5 \times 5 \mathrm{~mm}^{3}$ along the axes $<\overline{1} 10>\times<110>\times<001>$ respectively. As was also suggested previously[24, 25, 27, 28], the beam propagates along $<\overline{1} 10>$ axis and is polarized along $<110>$ axis. The crystal is thermally stabilized with a Peltier cell. Finally, a steady $10 \mathrm{kV} / \mathrm{cm}$ external electric field is applied along the $<001>$ axis: during all the experiments, the electric field is kept on and the laser beam is switched on and off using the accousto-optic modulator.

The goal of this experiment is to get an insight on the temporal behavior of the laser beam on the output face of the sample from the time the laser is switched on, without using an expensive high speed camera. To this aim, the acousto-optic modulator is modulated around the Hertz; thus, the beam is on for $500 \mathrm{~ms}$ and off for the next $500 \mathrm{~ms}$. The frequency is not of importance as long as the period (here around a second) is long enough so that the crystal has the time to reach its steady state after turning the beam on. Also, the frequency is low enough that the crystal has the time to fully relax in between two consecutive pulses.

The output face of the crystal sample is imaged on a high speed position detector, which is in fact, as shown on figure 1, a set of 4 photodiodes carefully adjusted to allow beam position tracking: by comparing the powers received on the 4 diodes, the beam center of gravity can be located. Furthermore, if an off-center beam focuses, the 4 powers measured by the position detector will change. Therefore, this setup allows us to monitor the self-bending and selffocusing of a beam. Only a centered focusing beam cannot be monitored. However, this last case is not likely, as previously found [25].

A typical measurement is shown on figure 2 where a beam deviates to the right, as shown in fig 1 (b). Figure $2(\mathrm{a})$ is a plot of the detector 4 signals without applied field. The measured powers are nearly equal and constant with time: the beam is centered on the detector and does not move. This measure serves as a reference. The fact (a)
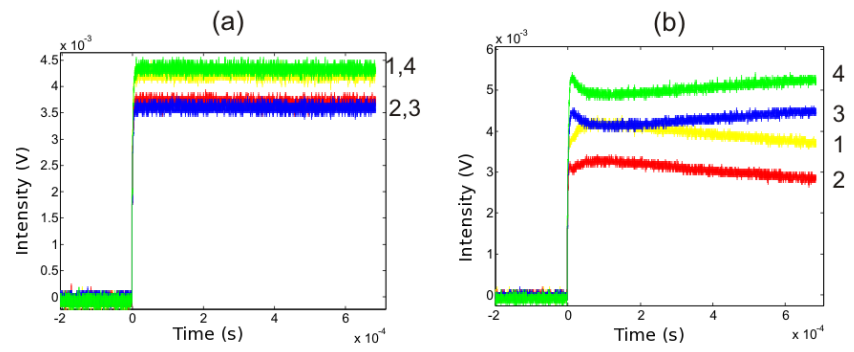

Figure 2: Typical measurement output from the position detector. Each curve shows the intensity measured by the quadrant of the same number and color as in figure 1, in the same conditions : (a) centered beam, (b) off-centered beam as in figure 1. The wavelength here is $1064 \mathrm{~nm}$. The temperature is $20^{\circ} \mathrm{C}$ and the beam intensity is $10 \mathrm{~W} / \mathrm{cm}^{2}$. (a)

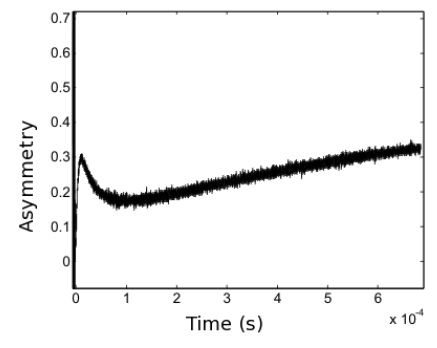

(b)

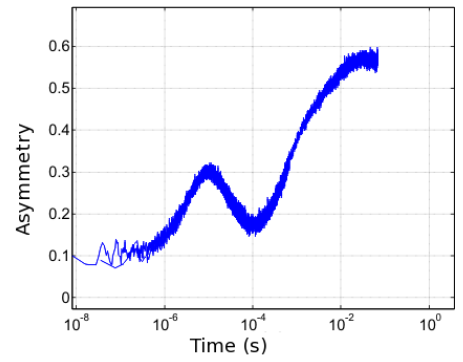

Figure 3: Asymmetry calculated by applying equation (1) to the measurements given in figure $2(\mathrm{~b})$, in a linear scale (a) and semilogarithmic (b).

that the 4 measured powers cannot be made equal by centering the beam more precisely on the detector is due to imperfections in the InP:Fe crystal, which alter slightly the Gaussian shape of the beam during propagation. A motion of the beam as the one that is shown on figure 1(b) leads to the 4 detected signals shown on figure $2(\mathrm{~b})$.

In order to make systematic measurements of the beam motion on the detector, we considered the fact, given from previous theoretical and experimental literature [24-28], that the beam bends mainly in the direction parallel to the externally applied electric field - direction which corresponds to the horizontal direction in figure 1 . We have therefore defined the asymmetry $\alpha$ as

$$
\alpha=1-\frac{I_{1}+I_{2}}{I_{3}+I_{4}}
$$

where $I_{n}$ is the optical power received on the photodiode numbered $n$ on figure 1 .

Thus, from the camera observation of steady state and the measurements issued from the detector, a time resolved measurement of the asymmetry can be deduced: for instance, the measurements shown in figure 2(b) lead to the asymmetry shown in 3 . As such, any variation of 

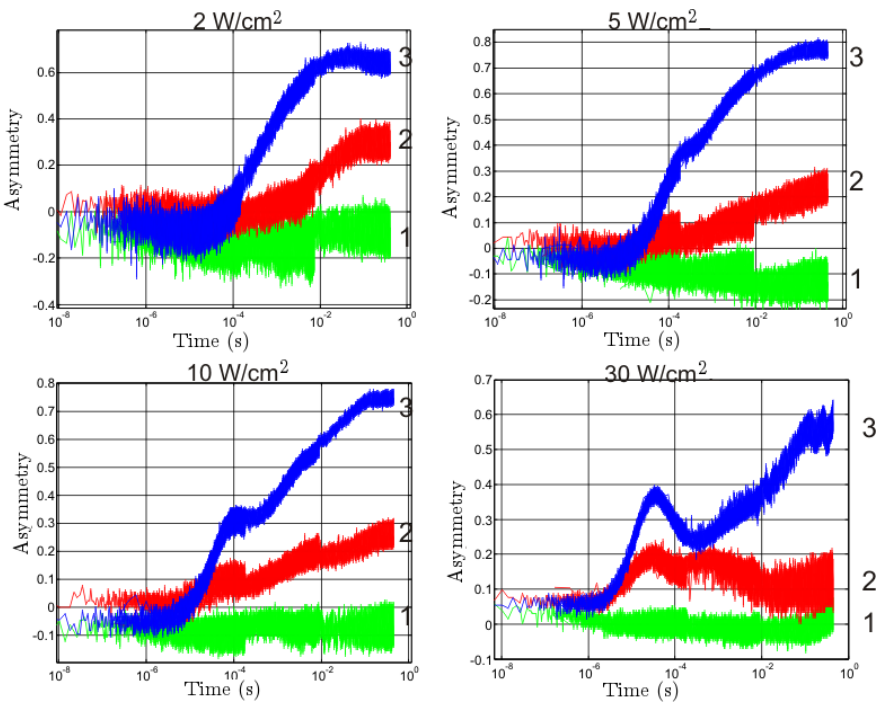

Figure 4: Time resolved asymmetry for several intensities of a 1.55 micron wavelength single beam, a background intensity of 0.4 $\mathrm{W} / \mathrm{cm}^{2}$, a $10^{\circ} \mathrm{C}$ temperature and an applied field of $10 \mathrm{kV} / \mathrm{cm}$ for the following set of experimental conditions : $(1-$ green - reference plot) electric field off, background intensity off, $(2-$ red $)$ electric field on, background intensity on, $(3$ - blue) electric field on, background intensity off. The apparent discontinuity that appears on some of the above graphs around the round values of the decades are in fact a discontinuity in the noise. This is due to the fact that these curves take advantage of a very reproducible phenomenon: they are the superposition of different measures for different calibers of the oscilloscope.

the asymmetry is due to a combination of beam bending and focusing.

Finally and to conclude this description of the experimental setup, let us mention that measurements with an homogeneous background intensity are also included in this analysis, to complete previous steady state measurements [26]. As has been shown before [14], the background intensity needs to be of the same order of magnitude as the signal beam peak intensity, implying the use of a laser source much more intense for the background intensity than for the signal. For this reason, these measurements will only be presented for the $1.55 \mu \mathrm{m}$ wavelength, the background intensity being provided by our $1064 \mathrm{Nd}$ :YAG laser, expanded to shine on the whole sample.

As can be seen on figure 3 , the asymmetry temporal behavior is characterized by two distinct time scales. The following sections will be devoted to the study of theses two time scales and the corresponding values of the asymmetry. The next section proposes a time resolved analysis of the asymmetry behavior as a function of beam and intensity and temperature, with and without background intensity. Section 4 provides a tentative interpretation of the results.

\section{Asymmetry time resolved behavior}

Figure 4 shows a set of asymmetry measurements at the $1.55 \mu \mathrm{m}$ wavelength. This figure is representative of a larger set of experiments, for beam intensities ranging from 0.1 to $90 \mathrm{~W} / \mathrm{cm}^{2}$. The measurements are presented for various intensities, with and without the background intensity.

The first observation is that the background intensity attenuates the asymmetry evolution, as the values measured are always lower when the background intensity is on. This was observed before, for steady state only[26]. The second, and probably more important, is that both the intensity of the beam and the background intensity have a strong influence on the very existence of the first time scale: the time to reach the first maximum rises if the beam intensity is lowered, whereas the second time scale does not seem to change. Therefore, the two time scales merge for low intensities. Finally, the presence of the first time scale is not obvious if the background intensity is present.

Figure 5 shows a similar set of experimental results at the wavelength of $1064 \mathrm{~nm}$, without background intensity. Similar conclusions can be drawn: the time scales merge for low intensities.

The same experiments can be conducted with a reverse field. We have done it at the $1064 \mathrm{~nm}$ wavelength and reported the most significant results on figure 6 . These latest measurements lead to an important observation: whereas the long time scale asymmetry changes its sign with the field, as could be foreseen from steady state beam bending[26-28], the short time scale asymmetry is not influenced by the sign of the applied electric field. This is doubtlessly the sign that two different mechanisms are operating on the beam bending at two time scales: the fastest and field independent one is probably linked to a space charge field building on the diffusion of free carriers, whereas the slow field-dependent one depends on carriers drifting in the electric field. Another hint at the nature of these two different mechanisms at work is the difference in the build-up time: the shorter time is inversely proportional to the beam intensity, whereas the longer one barely depends on it.

A further step towards the identification of the transport mechanisms at work is the study of the influence of temperature upon the temporal behavior of the asymmetry. The most significant results are given of figure 7 for a $1064 \mathrm{~nm}$ wavelength beam. Once again, the two time scales are retrieved. However, in this case, it the short time scale which is temperature independent, whereas the long one is strongly reduced when the temperature rises, leading again to their merging for moderately high temperature.

\section{Two distinct time scales}

The measurements presented in this paper evidence that the photorefractive effect in iron doped indium phosphide does occur on the microsecond range for moderate beam intensities around the $\mathrm{W} / \mathrm{cm}^{2}$. The measured asymmetry, which is closely related to the beam bending, evolves on two characteristic time scales, which are sensitive to the 

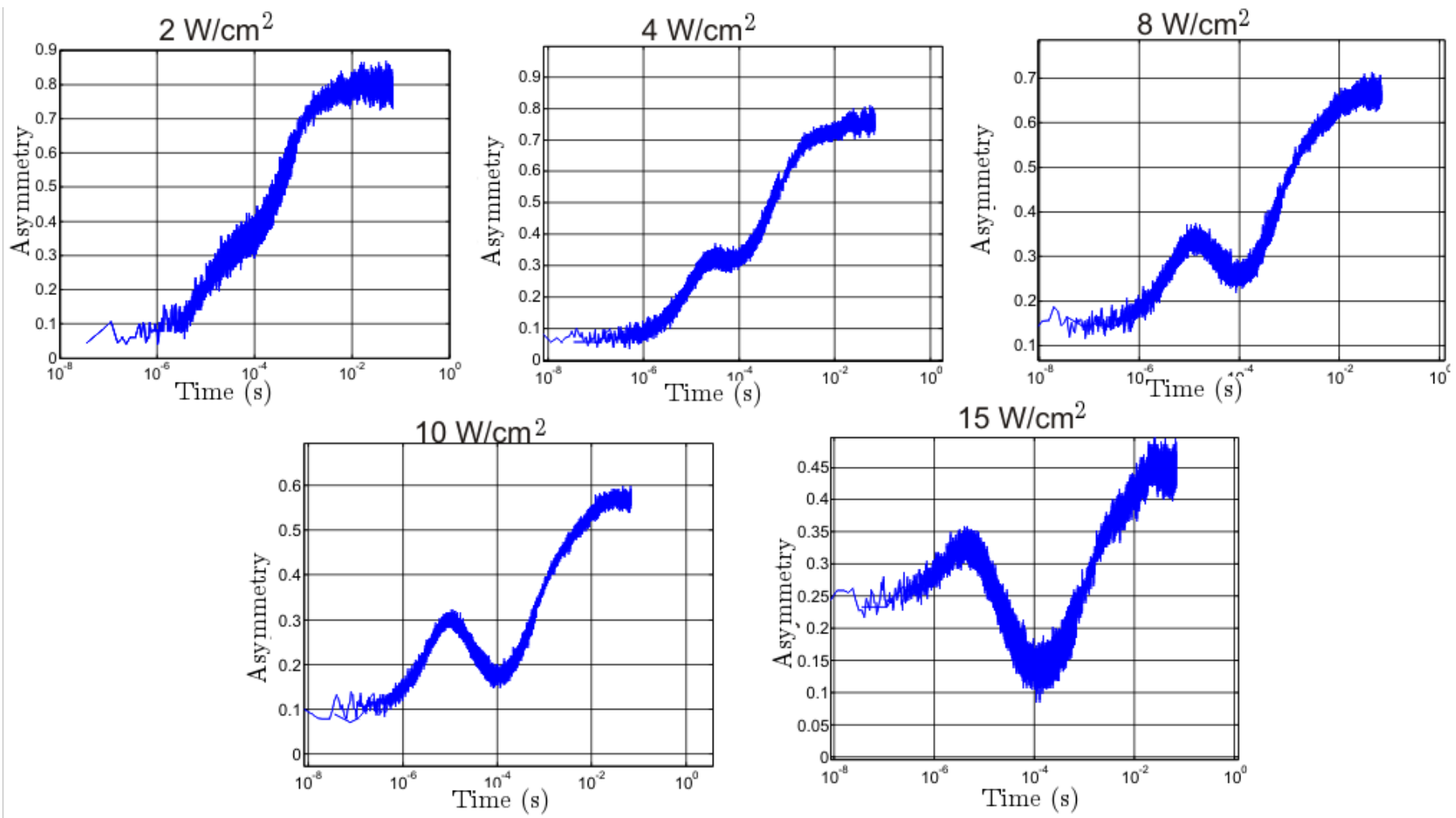

Figure 5: Time resolved asymmetry for several intensities of a 1.06 micron wavelength single beam, no background intensity, a $10^{\circ} \mathrm{C}$ temperature and an applied field of $10 \mathrm{kV} / \mathrm{cm}$

beam intensity and temperature, for the shorter one and the longer one respectively. On the contrary, the values reached by the asymmetry, close to 0.5 , do not exhibit a strong dependence on both intensity and temperature, apart from a tendency to decrease with increasing beam power (see figures 4 and 5).

The different behaviors of the two time scales are characteristic of two different photorefractive mechanisms: the short time scale is independent of applied field sign, decreases with increasing intensity, and slightly decreases with temperature. The long time scale is independent of intensity but changes sign with the applied field and decreases with increasing temperature. Both are influenced by a background irradiance. Their time dependence are plotted on figure 8 .

The influence of the background irradiance is a signature of the photorefractive nature of the observed phenomenon. The measurement we made were that of the asymmetry, as defined by equation (1). This characteristic does not allow a clear distinction between a bending of the beam and a self-focusing. However, it seems obvious that asymmetry is more affected by beam bending than by any other beam self action.

In this view, the long time scale can be interpreted as a drift associated phenomenon, as it changes sign with the electric field. Its decrease with increasing temperature is probably due to an increase with temperature of the den- sity of free carriers able to play a role in the photorefractive process. Its independence of intensity suggests that the free carriers involved are mostly thermally generated. In InP:Fe, these are known to be the electrons[19-22].

The short time scale independence of the electric field is a signature of a diffusion related process, which is known to lead to beam self-bending[14]. As for the long time scale, its slight decrease with temperature can be interpreted by an increase in the carrier density. Furthermore, its strong dependence on beam intensity suggest that photo-excited free carriers plays the major role. In InP:Fe, these are known to be the holes[19-22].

\section{Conclusion}

An experimental investigation of a single beam behavior in a electrically biased iron doped indium phosphide was conducted down to the microsecond scale. The photorefractive effect has been shown to be responsible for beam bending on the microsecond time scale. This conclusion results from the investigation of the time scales dependency on beam intensity, electric field direction and temperature.

However, the here reported experimental behavior of a single beam in Iron doped Indium Phosphide on such a short time had never been observed nor mentioned. Furthermore, it is neither predicted nor explained by the existing theoretical models. Evidencing and studying two time scales has allowed to hint that two different trans- 
$2 \mathrm{~W} / \mathrm{cm}^{2}$

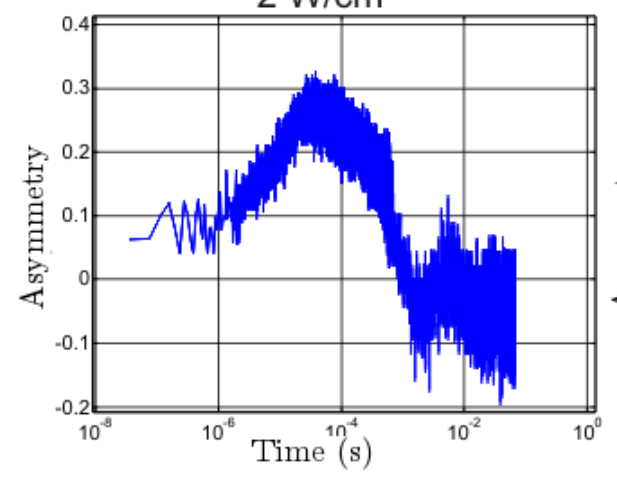

$4 \mathrm{~W} / \mathrm{cm}^{2}$

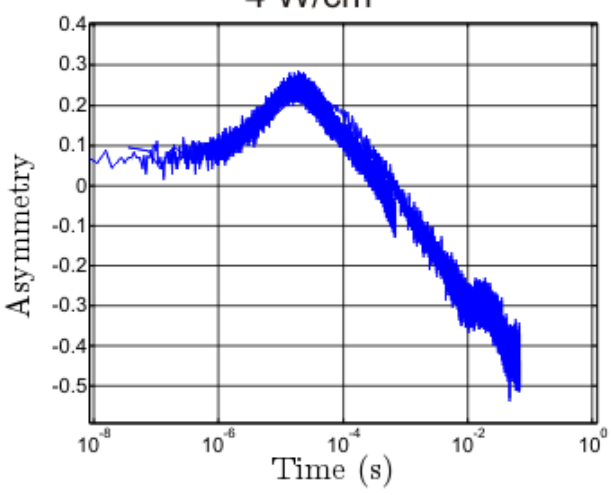

$8 \mathrm{~W} / \mathrm{cm}^{2}$

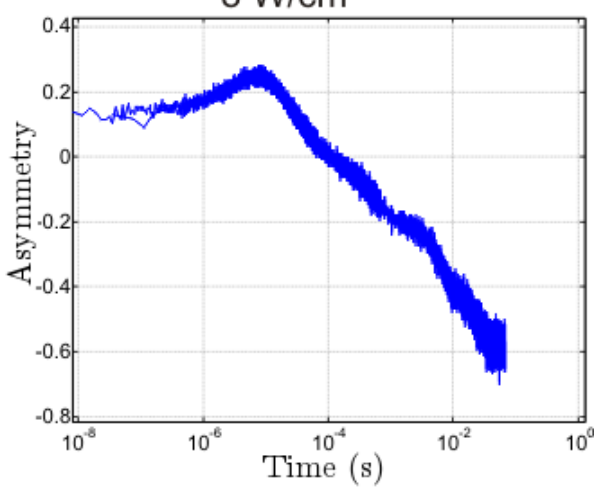

$15 \mathrm{~W} / \mathrm{cm}^{2}$
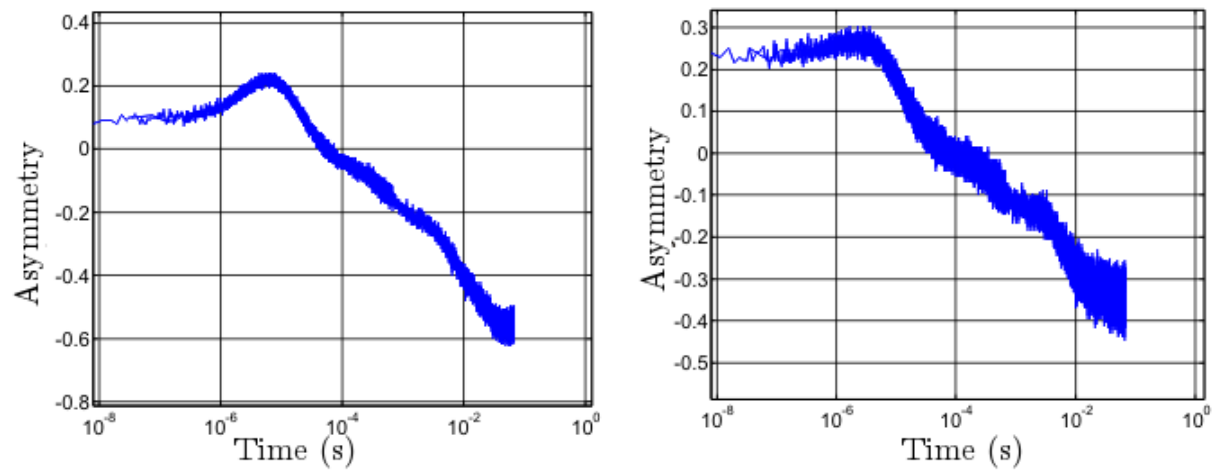

Figure 6: Time resolved asymmetry for several intensities of a 1.06 micron wavelength single beam, no background intensity, a $10^{\circ} \mathrm{C}$ temperature and an applied field of $-10 \mathrm{kV} / \mathrm{cm}$ : same conditions as in Fig 5 with opposite field. Let us note here that the sign of the electric field is arbitrary: we have chosen it to be positive when it mainly induces self-focusing, as was done before[26]. 


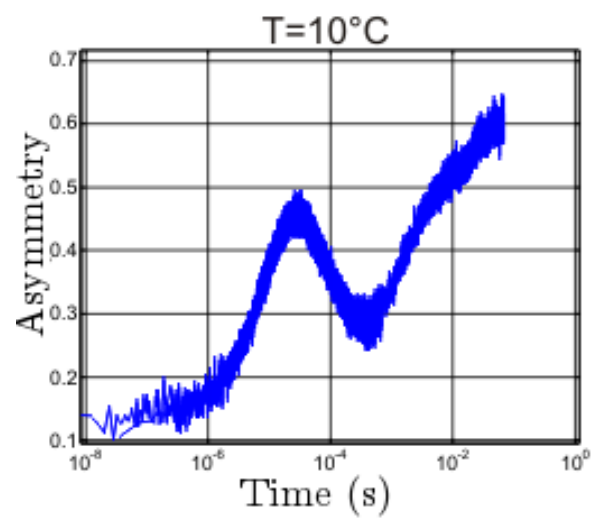

Time (s)
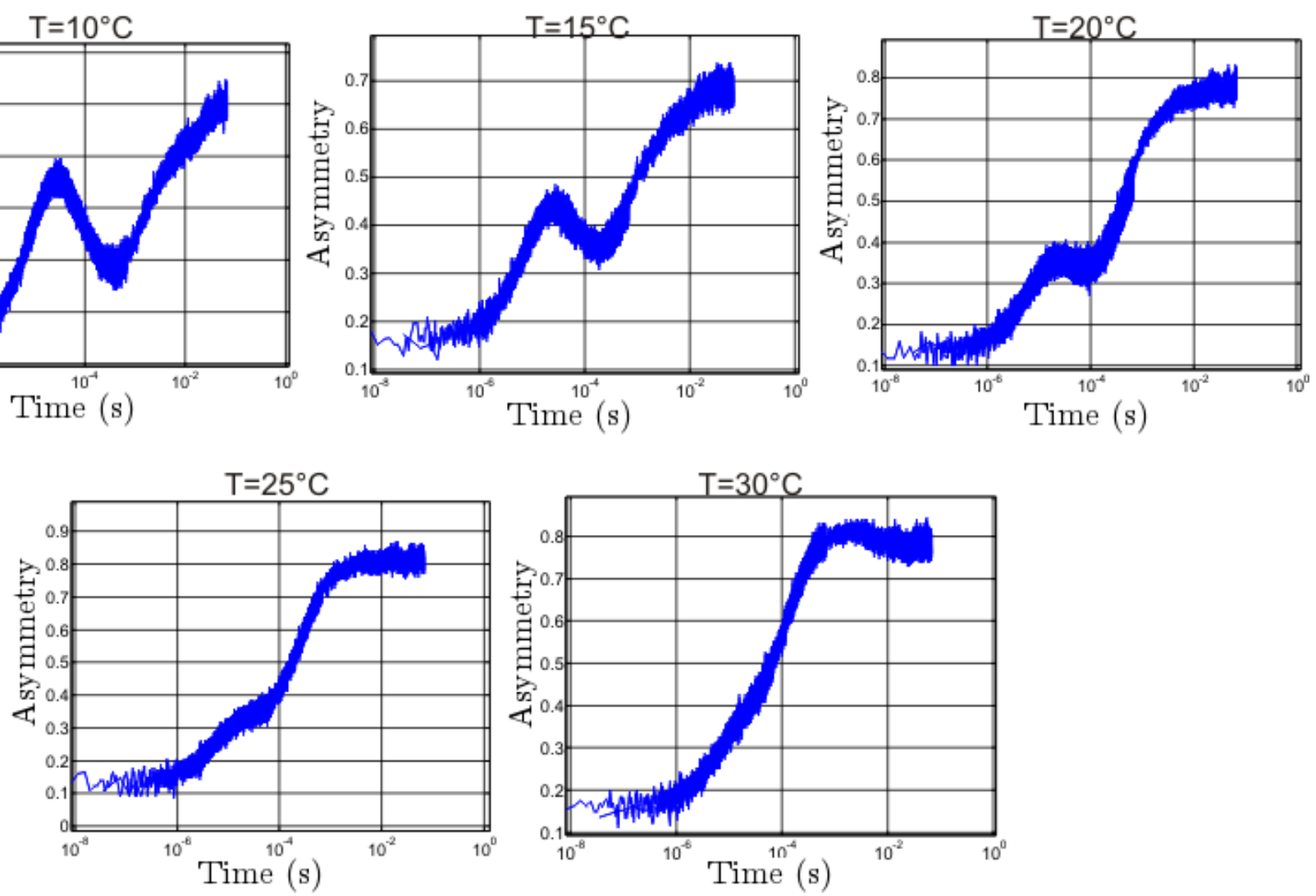

Figure 7: Time resolved asymmetry of a $1.06 \mu \mathrm{m} 3.2 \mathrm{~W} / \mathrm{cm}^{2}$ beam for several temperatures. The beam wavelength is $1064 \mathrm{~nm}$. Its intensity is $3.2 \mathrm{~W} / \mathrm{cm}^{2}$. The applied field is $10 \mathrm{kV} / \mathrm{cm}$.
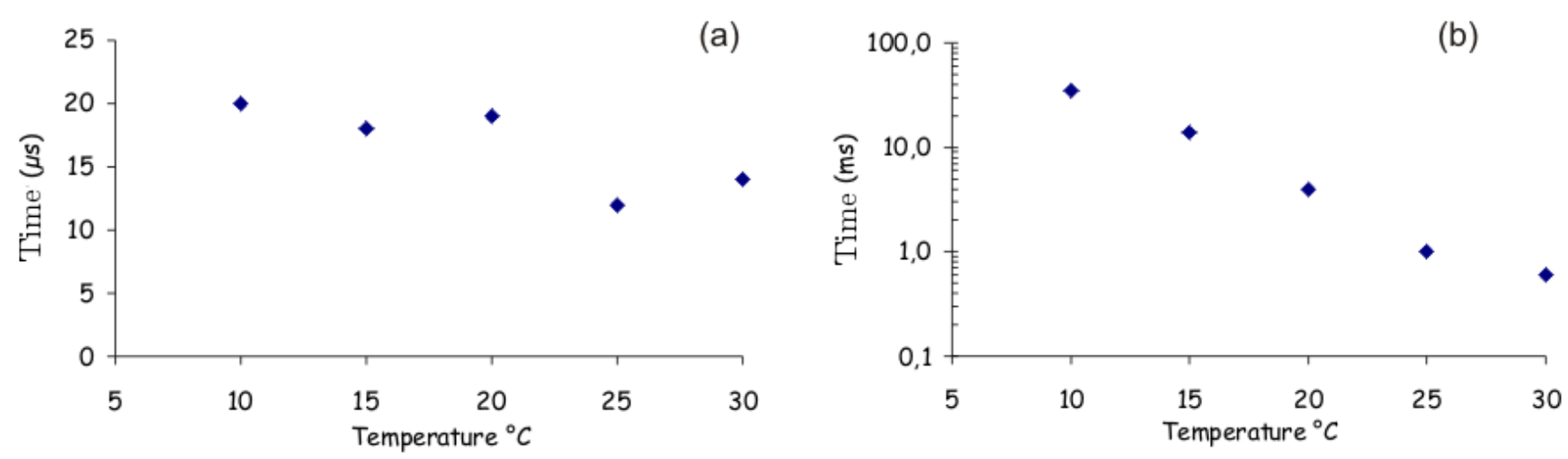

Figure 8: Time to reach the first maximum (a) and time to reach steady state (b). Experimental conditions are the same as in figure 7. 
port mechanism are at work, probably hole diffusion for the short time scale and electron drift for the long one.

\section{References}

[1] M. Segev, B. Crosignani, and A. Yariv. Spatial solitons in photorefractive media. Phys. Rev. Lett., 68(7):923, 1992.

[2] B. Crosignani, M. Segev, D. Engin, P. Di Porto, A. Yariv, and G. Salamo. Self-trapping of optical beams in photorefractive media. J. Opt. Soc. Am. B, 10(3):443, 031993.

[3] G. C. Duree, J. L. Shultz, G. J. Salamo, M. Segev, A. Yariv, B. Crosignani, P. Di Porto, and E. J. Sharp. Observation of selftrapping of an optical beam due to the photorefractive effect. Phys. Rev. Lett., 71(4):533, 061993.

[4] M. D. Iturbe-Castillo, P. A. Marquez-Aguilar, J. J. SanchezMandragon, S. Stepanov, and V. Vysloukh. Spatial solitons in photorefractive $\mathrm{Bi}_{12} \mathrm{TiO}_{20}$ with drift mechanism of nonlinearity. Appl. Phys. Lett., 64(4):408, 011994.

[5] K. Kos, H. Meng, G. Salamo, M. Shih, M. Segev, and G. C. Valley. One-dimensional steady state photorefractive screening soliton. Phys. Rev. B, 53(5):4330, 051996.

[6] M. Shih, M. Segev, G. C. Vally, G. Salamo, B. Crosignani, and P. DiPorto. Observation of two dimensional steady-state photorefractive screening solitons. Elec. Lett., 31:826-827, 1995.

[7] J. A. Andrade-Lucio, M. D. Iturbe-Castillo, P. A. MarquezAguilar, and R. Ramos-Garcia. Self-focusing in photorefractive $\mathrm{BaTiO}_{3}$. Optical and Quantum Electronics, 30:829-834, 1998.

[8] C. Astassiou, M. F. Shih, M. Mitchell, Z. Chen, and M. Segev. Optically induced photovoltaic self-focusing-to-self-defocusing transition. Opt. Lett., 23(12):924, 061998.

[9] S. R. Singh and D. N. Christodoulides. Evolution of spatial optical solitons in biased photorefractive media under steady state conditions. Opt. Commun., 118:569, 081995.

[10] A. A. Zozulya, D. Z. Anderson, A. V. Mamaev, and M. Saffman. Self-focusing and soliton formation in media with anisotropic nonlocal material response. Europhys. Lett., 36:419, 1996.

[11] A.A. Zozulya, D.Z. Anderson, A.V. Mamaev, and M.Saffman. Solitary attractors and low-order filamentation in anisotropic self-focusing media. Physical Review A, 57:522-534, 1998.

[12] M. Segev, M. Shih, and G. C. Valley. Photorefractive screening solitons of high and low intensity. Journ. Opt. Soc. Am. B, 13:706, 1996.

[13] A. A. Zozulya and D. Z. Anderson. Nonstationary self-focusing in photorefractive media. Opt. Lett., 20(8):837, 041995.

[14] N. Fressengeas, J. Maufoy, and G. Kugel. Temporal behavior of bi-dimensional photorefractive bright spatial solitons. Phys. Rev. E, 54:6866, 1996.

[15] C. Dan, D. Wolfersberger, N. Fressengeas, G. Montemezzani, and A. A. Grabar. Near infrared photorefractive self focusing in $\mathrm{Sn}_{2} \mathrm{P}_{2} \mathrm{~S}_{6}$ crystals. Optics Express, 15(20):12777, 2007.

[16] D. Wolfersberger, N. Fressengeas, J. Maufoy, and G. Kugel. Experimental self-focusing of a single laser pulse in $\mathrm{Bi}_{12} \mathrm{TiO}_{20}$. Journ. App. Phys., 89:2511, 2001.

[17] D. Wolfersberger, N. Khelfaoui, C. Dan, N. Fressengeas, and H. Leblond. Fast photorefractive self-focusing in InP:Fe semiconductor at infrared wavelengths. Appl. Phys. Lett., 92(2):21106-1-3, 2008.

[18] Massimo Alonzo, Cristian Dan, Delphine Wolfersberger, and Eugenio Fazio. Coherent collisions of infrared self-trapped beams in photorefractive InP:Fe. Applied Physics Letters, 96(12):121111-1-3, apr 2010.

[19] A. A-Idrissi, C. Ozkul, N. Wolffer, P. Gravey, and G. Picoli. Resonant behaviour of the temporal response of the two-wave mixing in photorefractive InP:Fe crystals under dc fields. Opt. Comm., 86:317-323, 1991.

[20] Bernard Mainguet. Characterization of the photorefractive effect in InP:Fe by using two-wave mixing under electric fields. Optics Letters, 13:657, 1988.

[21] G. Picoli, P. Gravey, and C. Ozkul. Model for resonant intensity dependence of photorefractive two-wave mixing in InP:Fe. Opt. Lett., 14(24):1362, 121989.
[22] G. Picoli, P. Gravey, C. Ozkul, and V. Vieux. Theory of twowave mixing gain enhancement in photorefractive InP:Fe : A new mecanism of resonance. Appl. Phys., 66:3798, 1989.

[23] Hervé Leblond and Nicolas Fressengeas. The theory of photorefractive resonance for localized beams in two-carrier photorefractive systems. Physical Review A: Atomic, Molecular and Optical Physics, 80(3):033837, September 2009.

[24] N. Fressengeas, N. Khelfaoui, C. Dan, D. Wolfersberger, G. Montemezzani, H. Leblond, and M. Chauvet. Roles of resonance and dark irradiance for infrared photorefractive selffocusing and solitons in bi-polar InP:Fe. Phys. Rev. A, 75:063834, 2007.

[25] F. Devaux and M. Chauvet. Three-dimensional numerical model of the dynamics of photorefractive beam self-focusing in InP:Fe. Phys. Rev. A, 79:033823, 2009.

[26] C. Dan, D. Wolfersberger, and N. Fressengeas. Experimental control of steady state photorefractive self-focusing in InP:Fe at infrared wavelengths. Applied Physics B: Lasers and Optics, pages 1-9, 2011. 10.1007/s00340-011-4438-y.

[27] M. Chauvet, S.A. Hawkins, G.J. Salamo, M. Segev, D.F. Bliss, and G. Bryant. Self-trapping of planar optical beams by use of the photorefractive effect in InP:Fe. Opt. Lett., 21(17):1333, 09 1996.

[28] M. Chauvet, S. A. Hawkins, G. J. Salamo, M. Segev, D. F. Bliss, and G. Bryant. Self trapping of two dimensional optical beams and light induced waveguiding in photorefractive InP at telecomunication wavelengths. Appl. Phys. Lett., 70:2499-2501, 1997. 